\title{
Comparison of one-site versus two-site phacotrabeculectomy without the use of antimetabolites intraoperatively in patients with pseudoexfoliation glaucoma and primary open-angle glaucoma
}

\section{Eleni Bagli \\ Christos Gartzios loannis Asproudis Georgios Kitsos}

University Eye Clinic of loannina, loannina, Greece
Correspondence: Eleni Bagli Aravantinou 6a, 45444 loannina, Greece Tel +30697449 I334

Fax +302651097888

Email elenibgl@hotmail.com
Purpose: The comparison of one-site versus two-site phacotrabeculectomy in patients with pseudoexfoliation glaucoma (PEXG) and primary open-angle glaucoma (POAG), with respect to intraocular pressure (IOP), antiglaucomatous medication (AM) requirements and visual acuity (VA).

Methods: Forty-seven patients (eyes) with cataract and POAG and 46 sex- and age-matched patients with cataract and PEXG were randomized to one- or two-site phacotrabeculectomy and reviewed with a follow-up at three years.

Results: Mean preoperative IOP was $22.04 \pm 2.27 \mathrm{mmHg}$ and $22.92 \pm 2.35 \mathrm{mmHg}$ in POAG and PEXG groups $(\mathrm{p}>0.05)$ using a mean of $2.76 \pm 0.74,2.74 \pm 0.69$ AM, respectively $(p>0.05)$. After three years of follow-up, the mean IOP was $15.04 \pm 1.57 \mathrm{mmHg}$ in the one-site, $15.04 \pm 1.99 \mathrm{mmHg}$ in the two-site group with POAG, $15 \pm 1.8 \mathrm{mmHg}$ in the one-site, $15.32 \pm 1.31 \mathrm{mmHg}$ in the two-site group with PEXG, using a mean of $0.68 \pm 0.69,0.84 \pm 0.75$, $0.96 \pm 0.67$, and $0.8 \pm 0.62 \mathrm{AM}$, respectively. Mean IOP and AM postoperatively were significantly less compared to preoperatively for each group $(\mathrm{p}<0.05)$. No difference was observed in mean IOP and AM between the four groups at three years postoperatively $(\mathrm{p}>0.05)$. VA improved similarly in four groups at the three-year follow-up $(\mathrm{p}<0.05)$. Intraoperative, postoperative complications, and need for 5 -fluorouracil injections were similar between the surgical groups. Conclusion: One-site and two-site phacotrabeculectomy without the use of antimetabolites intraoperatively were similarly safe and effective in IOP control over a three-year follow-up period in patients with POAG and PEXG.

Keywords: phacotrabeculectomy, pseudoexfoliation, glaucoma

\section{Introduction}

The concurrent existence of visually significant cataract and glaucoma is increasingly common in the elderly population. Because of this the ophthalmologist commonly faces a surgical decision on the best way to simultaneously manage these two conditions.

Combined cataract extraction, intraocular lens (IOL) implantation, and trabeculectomy are now widely accepted for managing cataracts in patients with poorly controlled glaucoma. In addition, the intraoperative and postoperative use of antimetabolites such as mitomycin $\mathrm{C}$ and 5-fluorouracil (5-FU) have been shown to produce lower intraocular pressure (IOP) after the operation. ${ }^{1-3}$

Phacotrabeculectomy is performed either with the two procedures (phacoemulsification and trabeculectomy) through the same incision (one site) or through separate incisions (two sites). 
It has been argued that the phacoemulcification incision separate from the trabeculectomy incision reduces postoperative scarring of the scleral flap and conjuctiva and improves the results of the filtration surgery. ${ }^{4}$

However, the decision to perform phacotrabeculectomy through one site or two sites remains controversial. Although outcomes in terms of visual acuity (VA) have been similar, ${ }^{5-8}$ there is a debate concerning which technique offers the best IOP control and reduction in glaucoma medication requirements. ${ }^{9-10}$

Pseudoexfoliation glaucoma (PEXG) has a high incidence in the elderly population. Because of this the coexistance of PEXG glaucoma and cataract is more likely. PEXG glaucoma is generally accepted as being more resistant to the treatment (surgical or medicational) compared to primary open-angle glaucoma (POAG). However there are no studies focusing on the comparison of one site to two-site combined phacotrabeculectomy technique in patients with PEXG.

The purpose of this study was to compare prospectively the results of one site with two-site combined phacoemulsification with IOL implantation and trabeculectomy surgery without the use of antimetabolites intraoperatively with respect to VA, IOP, and glaucoma medication requirements in patients with POAG and PEXG.

\section{Patients and methods}

The clinical outcomes of one-site and two-site phacotrabeculectomy were compared in a three-year prospective, randomized, controlled clinical trial. Patients were recruited and enrolled at the Glaucoma Unit of the University Eye Clinic of Ioannina, Greece. All operations were performed by one surgeon (GK).

\section{Patient enrollment and treatment assignment}

Criteria for inclusion in the study were the presence of cataract and glaucoma. All patients had a visually significant cataract that impaired some of their activities and they were also willing to have cataract extraction. The density of the cataract was similar among the patients. Moreover some of the patients had a decrease in their VA because of cataract ( $\geq 2$ lines by the Snellen method) the last preoperative months.

All patients had POAG or PEXG in the same eye with inadequate control of IOP or requiring two or more antiglaucoma medications. All eyes had to have had an IOP of more than $21 \mathrm{mmHg}$ on at least one previous examination. All patients had typical glaucoma visual defect by automated perimetry (Humphrey perimetry threshold 30.2 or 24.2) and also progression of glaucoma damage (perimetry testing and $\mathrm{C} / \mathrm{D}$ ratio evaluation) during the last months preoperatively. No ocular hypertensives were included in the study. All the patients had a minimum preoperative follow up of more than a year in the glaucoma unit of our clinic.

Exclusion criteria were the presence of neovascularization of the iris or angle, a history of uveitis, phacolytic or phacomorphic glaucoma, steroid-induced glaucoma, traumatic glaucoma, or previous incisional surgery in the same eye. Patients older than 89 years were not included. The authors did not enroll patients with co-existing ocular pathology that would affect final VA. Patients were randomized to one- or two-site phacotrabeculectomy without the use of antimetabolites intraoperatively, using a random numbers table. Finally patients with rupture of the posterior capsule during the phacoemulsification that leaded to insertion of anterior chamber IOL were also excluded from the study.

\section{Procedures}

All operated eyes received peribulbar anesthesia with 2\% lidocaine without epinephrine and $0.75 \%$ bupivacaine. Pupils were dilated with topical cyclopentolate $1 \%$ and phenylephrine $2.5 \%$.

\section{One-site surgery}

A 4-0 silk suture was placed under the superior rectus muscle and was used to rotate the globe inferiorly. A superior fornix-based conjunctival flap was made by incising the conjunctiva at the limbus and dissecting posteriorly. This flap was centered at 12 o'clock and was approximately $6 \mathrm{~mm}$ in chord length. Electrocautery was used to control episcleral bleeding. A limited tenonectomy was performed in most patients. A triangular partial-thickness scleral flap was mobilized superiorly. A paracentesis then was performed, followed by injection of a viscoelastic. A shelved entry into the anterior chamber under the scleral flap was made using a 3.2-mm blade keratome. Phacoemulsification then was performed. After cortical aspiration and reformation of the anterior chamber with viscoelastic, the wound was enlarged slightly with a crescent knife. A three-piece foldable acrylic intraocular lens was inserted into the capsular bag. After aspiration of all viscoelastic and injection of acetylcholine to constrict the pupil, a Descemet punch was used to create a $1.0-\mathrm{mm}$ diameter sclerostomy under the scleral flap. A small basal iridectomy was performed in all cases and a 10-0 nylon suture was placed at the apex of the flap and looped aside. 
The conjunctival incision was closed by passing sutures of 8-0 silk into limbal cornea at 10 o'clock and 2 o'clock. The anterior edge of the conjunctiva was advanced at least $1 \mathrm{~mm}$ over the cornea to decrease the risk of leakage.

Anterior chamber was deepened with a balanced salt solution through the paracentesis with good bleb elevation and no bleb leakage.

Antibiotic injection was performed intracamelar and a subconjunctival injection of dexamethasone was performed inferiorly. Drops of and pilocarpine $1 \%$ were applied to the cornea before placement of a patch and shield. A single 500-mg dose of oral acetazolamide was given in the recovery room to every patient unless a sulfa allergy was documented.

\section{Two-site surgery}

Two-site surgery began with the surgeon seated temporally. A paracentesis was made, followed by injection of viscoelastic. A temporal clear corneal incision was made with a 3.2-mm blade keratome. Phacoemulsification and intraocular lens implantation then was performed as described for the one-site surgery. The surgeon then switched position and was seated superiorly for the trabeculectomy. A 4-0 silk suture was passed through superior corneal tissue to rotate the globe downward and laterally. A fornix-based conjunctival flap was created and a limited tenonectomy was performed in most cases. After applying light electrocautery and exposing the conjunctival insertion anteriorly at the limbus, a triangular scleral flap was fashioned as described for the one-site surgery.

With a crescent knife under the scleral flap, the dissection then was advanced into clear cornea. The anterior chamber was entered with a 3.2-mm blade keratome under the anterior hinge of the scleral flap. The sclerostomy, iridectomy, and scleral flap closure were performed in the same manner as for the one-site surgery. The conjunctival wound was closed with $8-0$ silk suture. Injections and topical medications then were administered as with the one-site eyes.

The postoperative regimen for both groups of eyes was identical.

A topical antibiotic and were given three times daily for two weeks. Topical corticosteroids and nonsteroidal antiinflammatory medication were used three times daily for the first month, reduced gradually to two times daily for two weeks and then were tapered slowly. Adjunctive topical and oral antiglaucoma medications were used as needed for the first one or two weeks.

\section{Data collection}

Baseline data including IOP by Goldmann applanation tonometry and the number of topical and oral antiglaucoma medications used by the patient were recorded before surgery. The timolol and dorzolamide combination was considered to be two medications. Best-corrected VA was measured by the Snellen method. Other information collected before surgery included age, gender, diagnostic type of glaucoma, and history of previous laser trabeculoplasty or iridotomy. Data collected during surgery included operative time and complications.

Data were collected at each postoperative visit (included IOP, number of supplemental antiglaucoma medications, best-corrected VA, occurrence and nature of any complications) by two different ophthalmologists of the glaucoma unit, who did not know the preoperative clinical record of the patients. All the data (preoperative and postoperative) were analyzed at the end of the follow up.

Five subconjuctival injections of 5-FU (0.1 ml$50 \mathrm{mg} / \mathrm{ml}$ ) were performed into the inferior fornix daily when IOP was higher than $18 \mathrm{mmHg}$ between 2-3 weeks postoperatively.

SPSS version 14.0 (SPSS Inc., Chicago, IL) was used for the statistical analysis and $p$ value less than 0.05 was considered as statistical significant.

\section{Results}

One hundred patients (50 patients with POAG-group1 and 50 patients with PEXG-group 2) met the initial inclusion criteria of the study. One eye of each subject included in the study was randomized to one of the two procedures and underwent surgery according to the study protocol. A total of seven patients were excluded from the study because of intra-operative rupture of posterior capsule and insertion of anterior chamber intraocular lens (Table 1). As a result, ninety-three patients (47 patients with POAG-group1 and 46 patients with PEXG-group 2) were finally enrolled in the study. The disposition of all study subjects and the baseline characteristics of each group are summarized in Table 2 and confirm that the two groups were similar in age, gender, and cup/disc ratio.

Mean IOP, number of medications, and VA were similar in POAG and PEXG groups preoperatively (Table 3).

In the POAG group there was no statistically significant difference between the one-site (subgroup 1A) and two-site (subgroup 1B) phacotrabeculectomy subgroups, in the mean IOP before surgery ( $p=0.627$; Mann-Whitney test). There was also no significant difference in IOP at all postoperative time points including the last follow up (36 months) between the two groups, $(\mathrm{p}=0.160$; Mann-Whitney test) (Figure 1a). However, both groups had statistically significantly less IOP 
Table I Intra- and postoperative complications in one-site and two-site phacotrabeculectomy

\begin{tabular}{|c|c|c|c|c|}
\hline & \multicolumn{2}{|l|}{ POAG (50) } & \multicolumn{2}{|l|}{ PEXG (50) } \\
\hline & One-site (IA) & Two-site (I B) & One-site (2A) & Two-site (2B) \\
\hline Capsular rupture-AC IOL & $\mathrm{I}(4 \%)$ & $2(8 \%)$ & $2(8 \%)$ & $2(8 \%)$ \\
\hline Capsular rupture-PC IOL & $2(8.3 \%)$ & I (4.3\%) & $2(8.7 \%)$ & $2(8.7 \%)$ \\
\hline $\begin{array}{l}\text { Conjuctival bleb leaks Early } \\
\text { ( }<7 \text { days) }\end{array}$ & $2(8.3 \%)$ & $3(13 \%)$ & $2(8.7 \%)$ & $3(13 \%)$ \\
\hline $\begin{array}{l}\text { Grade I Spaeth shallow } \\
\text { anterior chamber^ }\end{array}$ & 0 & 0 & I (4.3\%) & I (4.3\%) \\
\hline $\begin{array}{l}\text { Grade } 2 \text { Spaeth shallow } \\
\text { anterior chamber^ }\end{array}$ & I (4.2\%) & I (4.3\%) & $2(8.7 \%)$ & I (4.3\%) \\
\hline Choroidal effusion & I (4\%) & I (4.3\%) & I (4.3\%) & I (4.3\%) \\
\hline 5-FU & $8(33 \%)$ & $7(30 \%)$ & $12(52 \%)$ & II (48\%) \\
\hline Bleb encapsulation & I (4\%) & I (4.3\%) & 0 & I (4.3\%) \\
\hline N:YAG capsulotomy & $6(25 \%)$ & $4(17.4 \%)$ & 7 (30.4\%) & $5(21.7 \%)$ \\
\hline
\end{tabular}

Abbreviations: AC IOL, anterior chamber IOL; IOL, intraocular lens; PC IOL, posterior chamber IOL; PEXG, pseudoexfoliation glaucoma; POAG, primary open-angle glaucoma; 5-FU, 5-Fluorouracil.

Note: ${ }^{\wedge}$ Spaeth grading of shallow anterior chamber.

postoperatively at 36 months follow-up compared to baseline $(p=0.020$ and $p=0.023$, respectively; Table 4$)$. Mean number of supplemental antiglaucoma medications were analogous between subgroups $1 \mathrm{~A}$ and $1 \mathrm{~B}$ preoperatively $(\mathrm{p}=0.826$; Mann-Whitney test). There was also no statistical significant difference in number of medication between subgroups $1 \mathrm{~A}$ and $1 \mathrm{~B}$ during the follow-up (Table 5). However both groups needed less medication at 36 months postoperatively compared to baseline ( $p=0.031, p=0.032$, respectively; Table 4).

Similarly, in PEXG patients there was no statistical significant difference between the one-site (subgroup 2A) and two-site (subgroup 2B) phacotrabeculectomy subgroups concerning mean IOP preoperatively ( $\mathrm{p}=0.491$; MannWhitney test) and at any interval postoperatively including the last follow up ( $p=0.216$; Mann-Whitney test $)$ (Figure 1b). However, mean IOP was statistical significantly less at 36 months postoperatively compared to baseline for each subgroup ( $p=0.021, p=0.022$, respectively; Table 4).
The mean number of supplemental antiglaucoma medications needed in both subgroups (2A, 2B) in the PEXG group before surgery and during follow-up were also similar (Table 5). However, each group had a significant reduction in the requirement for antiglaucomatous medication postoperatively compared to preoperatively $(p=0.039, p=0.031$, respectively; Table 4).

The mean IOP was also similar between subgroups $1 \mathrm{~A}$ and 2A preoperatively and during follow up (Figure 2a). The same pattern of IOP variation was observed, when we compared subgroups 1B and 2B (Figure 2b).

Overall, the mean IOP and the number of antiglaucomatous medications at 36 months postoperatively were not statistically different between the four surgical groups $(p=0.902$ and $p=0.553$, respectively; Table 3$)$.

The number of patients not requiring antiglaucomatous medication after 36 months follow-up was higher for POAG patients (independent of the type of operation) compared

Table 2 Baseline characteristics for groups treated with one-site or two-site phacotrabeculectomy

\begin{tabular}{|c|c|c|c|c|c|}
\hline & \multicolumn{2}{|l|}{ POAG } & \multicolumn{2}{|l|}{ PEXG } & \multirow[t]{2}{*}{$\mathbf{p}$} \\
\hline & One-site (IA) & Two-site (IB) & One-site (2A) & Two-site (2B) & \\
\hline No of patients-eyes & 24 & 23 & 23 & 23 & \\
\hline Mean age (years) & $75.2 \pm 4.6$ & $76.3 \pm 5.4$ & $74.8 \pm 5.3$ & $74.9 \pm 4.6$ & $0.88 I$ \\
\hline Range (years) & $67-85$ & $68-85$ & $65-89$ & $67-85$ & \\
\hline Males/Females & $11 / 13$ & $10 / 13$ & $11 / 12$ & $10 / 13$ & $0.989 *$ \\
\hline Mean C/D ratio & $0.66 \pm 0.17$ & $0.68 \pm 0.15$ & $0.66 \pm 0.17$ & $0.68 \pm 0.16$ & 0.892 \\
\hline
\end{tabular}

Abbreviations: ANOVA, analysis of variance; C/D, cup/disc; PEXG, pseudoexfoliation glaucoma; POAG, primary open-angle glaucoma. Notes: One way ANOVA was performed; ${ }^{*}$ Chi square test. 
Table 3 Mean IOP, antiglaucoma medication,VA preoperatively and 36 months postoperatively

\begin{tabular}{|c|c|c|c|c|c|}
\hline & \multicolumn{2}{|l|}{ POAG } & \multicolumn{2}{|l|}{ PEXG } & \multirow[t]{2}{*}{$\mathbf{p}$} \\
\hline & One-site (IA) & Two-site (I B) & One-site (2A) & Two-site (2B) & \\
\hline IOP preop & $22.72 \pm 2.17 \mathrm{mmHg}$ & $21.36 \pm 2.37 \mathrm{mmHg}$ & $22.96 \pm 2.30 \mathrm{mmHg}$ & $22.92 \pm 2.45 \mathrm{mmHg}$ & 0.823 \\
\hline IOP postop & $15.04 \pm 1.57 \mathrm{mmHg}$ & $15.04 \pm 1.99 \mathrm{mmHg}$ & $15 \pm 1.80 \mathrm{mmHg}$ & $15.32 \pm 1.31 \mathrm{mmHg}$ & 0.902 \\
\hline AM preop & $2.84 \pm 0.69$ & $2.72 \pm 0.79$ & $2.68 \pm 0.69$ & $2.80 \pm 0.70$ & 0.546 \\
\hline AM postop & $0.68 \pm 0.69$ & $0.84 \pm 0.75$ & $0.96 \pm 0.67$ & $0.84 \pm 0.62$ & 0.553 \\
\hline VA preop & $0.26 \pm 0.16$ & $0.26 \pm 0.17$ & $0.23 \pm 0.14$ & $0.25 \pm 0.14$ & 0.882 \\
\hline VA postop & $0.60 \pm 0.31$ & $0.47 \pm 0.28$ & $0.56 \pm 0.26$ & $0.60 \pm 0.25$ & 0.265 \\
\hline
\end{tabular}

Abbreviations: IOP preop, mean intraocular pressure preoperatively; IOP postop, mean intraocular pressure postoperatively; AM preop, mean antiglaucoma medication preoperatively; AM postop, mean antiglaucoma medication postoperatively; PEXG, pseudoexfoliation glaucoma; POAG, primary open-angle glaucoma; VA preop, mean visual acuity preoperatively; VA postop, mean visual acuity postoperatively.

Note: One way ANOVA was performed.

to PEXG but this result was not statistically significant $(\mathrm{p}=0.150$; Table 6$)$.

Concerning the secondary outcome measures between groups the VA improved postoperatively compared to preoperatively in POAG and PEXG patients who underwent either one- or two-site phacotrabeculectomy (Table 4). However there was no statistical difference in the final postoperative VA between the four subgroups $(p=0.265$; Table 3).

There were few complications observed intra-operatively and during the follow-up period in the four surgical subgroups (Table 1). Laser suturelysis was not performed on any patients. One patient from each subgroup (a total of four) had a second trabeculectomy for uncontrolled IOP. The second operation took place between 18 and 24 months postoperatively. Twelve patients with POAG (six from subgroup 1A, six from subgroup 1B) and 12 patients with PEXG (seven from subgroup $2 \mathrm{~A}$, five from subgroup $2 \mathrm{~B}$ ) underwent laser capsulotomy ( $p=0.257$; Chi square test $)$ at $13.2 \pm 7.4$ and $12.8 \pm 6.5$ months after the phacotrabeculectomy, respectively. The number of eyes receiving 5-FU injections postoperatively was less for POAG patients (with either onesite or two-site phacotrabeculectomy) compared to PEXG patients but this difference was not statistically significant $(\mathrm{p}=0.607$; Chi square test $)$.

The mean duration of operation was less for one-site phacotrabeculectomy compared to two-site phacotrabeculectomy by $9 \pm 6 \mathrm{~min}$ for both glaucoma groups.

\section{Discussion}

Our study focused on the effect of one-site versus two-site fornix-based phacotrabeculectomy without the use of antimetabolites intraoperatively in two groups of glaucoma patients (POAG and PEXG).

Overall, no significant differences were observed in the main outcome measures of IOP and the need for supplemental

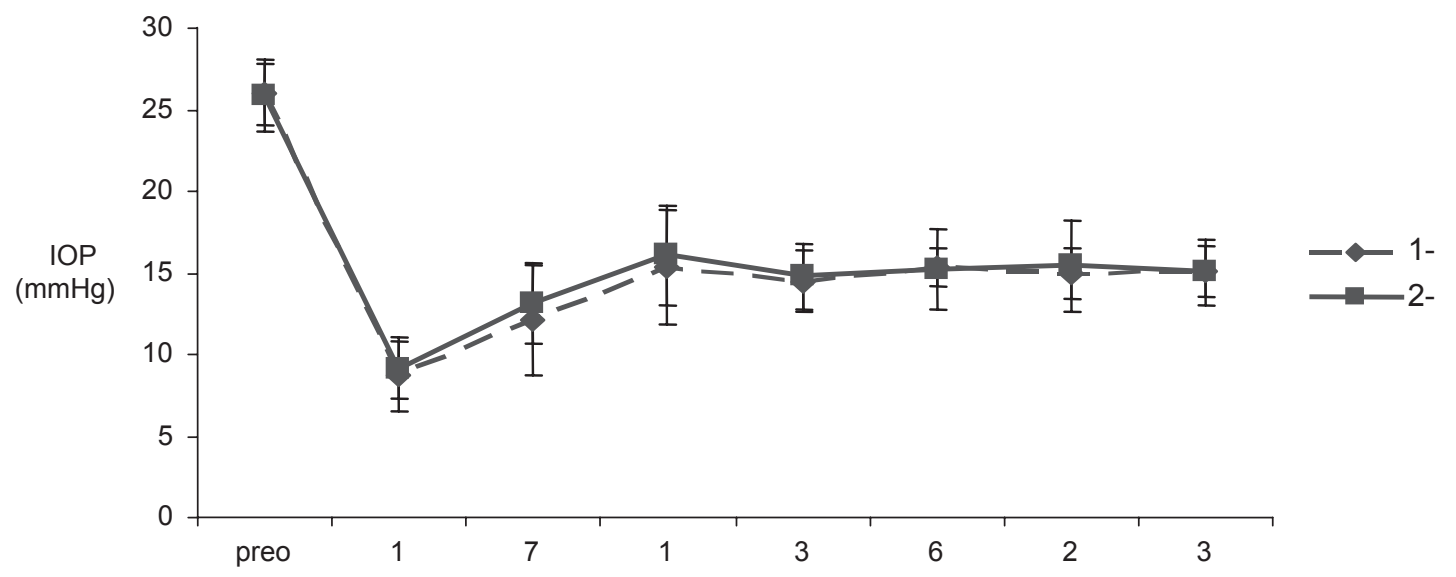

Figure Ia POAG group: Mean IOPs in the one-site and two-site phacotrabeculectomy groups at all time points. Abbreviations: IOP, intraocular pressure; PEXG, pseudoexfoliation glaucoma; POAG, primary open-angle glaucoma. 
Table 4 Mean IOP, number of antiglaucoma medications,VA preoperatively and 36 months postoperatively in the one-site and two-site phacotrabeculectomy in POAG and PEXG patients

\begin{tabular}{|c|c|c|c|c|c|c|c|c|}
\hline \multirow[b]{3}{*}{ IOP preop } & \multicolumn{4}{|l|}{ POAG } & \multicolumn{4}{|l|}{ PEXG } \\
\hline & \multicolumn{2}{|l|}{ One-site (IA) p I } & \multicolumn{2}{|l|}{ Two-site (I B) p2 } & \multicolumn{2}{|l|}{ One-site (2A) p3 } & \multicolumn{2}{|l|}{ Two-site (2B) p4 } \\
\hline & $22.72 \pm 2.17 \mathrm{mmHg}$ & 0.020 & $21.36 \pm 2.37 \mathrm{mmHg}$ & 0.023 & $22.96 \pm 2.30 \mathrm{mmHg}$ & 0.021 & $22.92 \pm 2.45 \mathrm{mmHg}$ & 0.022 \\
\hline IOP postop & $15.04 \pm 1.57 \mathrm{mmHg}$ & & $15.04 \pm 1.99 \mathrm{mmHg}$ & & $15 \pm 1.80 \mathrm{mmHg}$ & & $15.32 \pm 1.31 \mathrm{mmHg}$ & \\
\hline AM preop & $2.84 \pm 0.69$ & 0.031 & $2.72 \pm 0.79$ & 0.032 & $2.68 \pm 0.69$ & 0.039 & $2.80 \pm 0.70$ & 0.031 \\
\hline AM postop & $0.68 \pm 0.69$ & & $0.84 \pm 0.75$ & & $0.96 \pm 0.67$ & & $0.84 \pm 0.62$ & \\
\hline VA preop & 0.260 .16 & 0.001 & 0.260 .17 & 0.003 & 0.230 .14 & 0.001 & 0.250 .14 & 0.001 \\
\hline VA postop & 0.600 .31 & & 0.470 .28 & & 0.560 .26 & & 0.600 .25 & \\
\hline
\end{tabular}

Abbreviations: IOP preop, mean intraocular pressure preoperatively; IOP postop, mean intraocular pressure postoperatively; AM preop, mean antiglaucoma medication preoperatively; AM postop, mean antiglaucoma medication postoperatively; PEXG, pseudoexfoliation glaucoma; POAG, primary open-angle glaucoma; VA preop, mean visual acuity preoperatively; VA postop, mean visual acuity postoperatively.

Notes: PI, (significance) comparison in the one-site-POAG group: IOP preop vs IOP postop, AM preop vs AM postop, VA preop vs VA postop; p2 (significance) comparison in the two-site POAG group: IOP preop vs IOP postop, AM preop vs AM postop,VA preop vs VA postop; $\mathrm{p} 3$ (significance) comparison in the one-site PEXG group: IOP preop vs IOP postop,AM preop vs AM postop,VA preop vs VA postop; $\mathrm{p} 4$ (significance) comparison in the two-site PEXG group: IOP preop vs IOP postop, AM preop vs AM postop, VA preop vs VA postop; Mann-Whitney test.

antiglaucoma medications between the two glaucoma groups. Both surgical strategies were effective in reducing IOP and the mean IOP levels remained stable during the three years of follow-up. Both procedures were also able to eliminate the need for antiglaucoma medications in many eyes over the entire study period. These results suggest that bleb function was maintained in a high proportion of all operated eyes.

Two studies comparing one- versus two-site phacotrabeculectomy found that at the end of follow-up the one-site group required significantly more medications than the two-site group. ${ }^{9,10}$ In contrast, two other prospective studies and one retrospective study concluded, similarly to our study, that there was no statistical difference in the postoperative mean number of glaucoma medications or number of patients on glaucoma therapy in one- versus two-site phacotrabeculectomy. ${ }^{5,7,11}$

This study is unique in having compared the outcome of combined phacotrabeculectomy specifically in patients with PEXG. To the best of our knowledge, our study is the first to analyze separately the outcome of one- versus two-site phacotrabeculectomy in PEXG, a type of glaucoma known to be more resistant to medical and surgical therapy.

According to our results, the two types of combined procedures without the use of antimetabolites intra-operatively seem to be equivalent and successful in PEXG patients and the outcome is similar to patients with POAG. These findings are consistent with a previous report, where no difference was found in the outcome when combined phacotrabeculectomy

Table 5 Mean number of antiglaucoma medications in the one-site and two-site phacotrabeculectomy groups at all time points in POAG and PEXG patients

\begin{tabular}{|c|c|c|c|c|c|}
\hline \multirow[t]{2}{*}{$\overline{A M}$} & \multicolumn{2}{|l|}{ POAG } & \multicolumn{2}{|l|}{ PEXG } & \multirow[t]{2}{*}{$\mathbf{p}$} \\
\hline & One-site (IA) & Two-site (IB) & One-site (2A) & Two-site (2B) & \\
\hline Preop & $2.84 \pm 0.69$ & $2.72 \pm 0.80$ & $2.68 \pm 0.69$ & $2.80 \pm 0.70$ & 0.856 \\
\hline One day postop & 0 & 0 & 0 & 0 & \\
\hline Seven days postop & 0 & 0 & 0 & 0 & \\
\hline One month postop & 0 & $0.12 \pm 0.33$ & $0.20 \pm 0.40$ & $0.16 \pm 0.37$ & 0.387 \\
\hline Three months postop & 0 & $0.16 \pm 0.37$ & $0.16 \pm 0.37$ & $0.16 \pm 0.37$ & 0.973 \\
\hline Six months postop & $0.36 \pm 0.57$ & $0.64 \pm 0.63$ & $0.40 \pm 0.58$ & $0.26 \pm 0.37$ & 0.051 \\
\hline Two years postop & $0.64 \pm 0.70$ & $0.84 \pm 0.80$ & $0.88 \pm 0.66$ & $0.84 \pm 0.55$ & 0.601 \\
\hline Three years postop & $0.68 \pm 0.70$ & $0.84 \pm 0.75$ & $0.96 \pm 0.67$ & $0.84 \pm 0.62$ & 0.553 \\
\hline
\end{tabular}

Abbreviations: AM, antiglaucoma medication;ANOVA, analysis of variance; PEXG, pseudoexfoliation glaucoma; POAG, primary open-angle glaucoma; preop, preoperatively; postop, postoperatively.

Note: One way ANOVA was performed. 


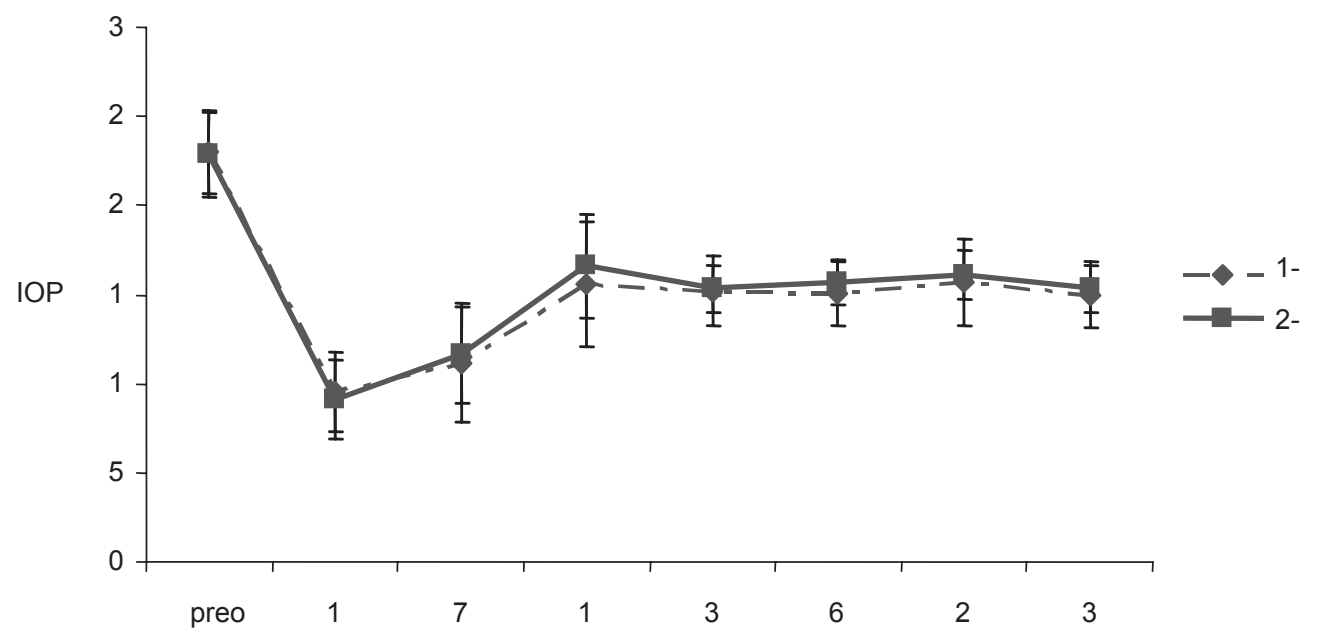

Figure Ib PEXG group: Mean IOPs in the one-site and two-site phacotrabeculectomy groups at all time points.

Abbreviations: IOP, intraocular pressure; PEXG, pseudoexfoliation glaucoma; POAG, primary open-angle glaucoma.

was performed in patients with POAG and PEXG. However, in that study mitomycin $\mathrm{C}$ was used intraoperatively and only one-site surgery was performed. ${ }^{12}$

In the current study we did find that two-site surgery took statistically significantly more time, involving changing the position and readjusting the microscope, than one-site surgery in both glaucoma groups and this is in accordance with other studies. ${ }^{5,13}$

Concerning the intraoperative and postoperative complications, they were few and relatively similar in oneand two-site phacotrabeculectomy for both POAG and PEXG. This result is in accordance with a previous report, where there was no significant difference in the rate of intraoperative and postoperative complications in one-site combined phacotrabeculectomy between PEXG and non-PEXG eyes, although the transition rate to extracapsular cataract extraction in eyes with PEXG was found to be higher (but not statistically significant). ${ }^{12}$

Although the two procedures seem to be equivalent in terms of the IOP control, reduction in need for antiglaucoma medications and improvement in VA, there were some differences noted between the two glaucoma groups. Fewer patients with POAG (27 patients) required antiglaucoma medication at the end of the follow-up compared to patients with PEXG (33 patients), although this result was not statistically significant $(\mathrm{p}>0.05)$. We expected this result to be statistically significant since PEXG is considered as unpredictable and resistant to therapy glaucoma. However, more

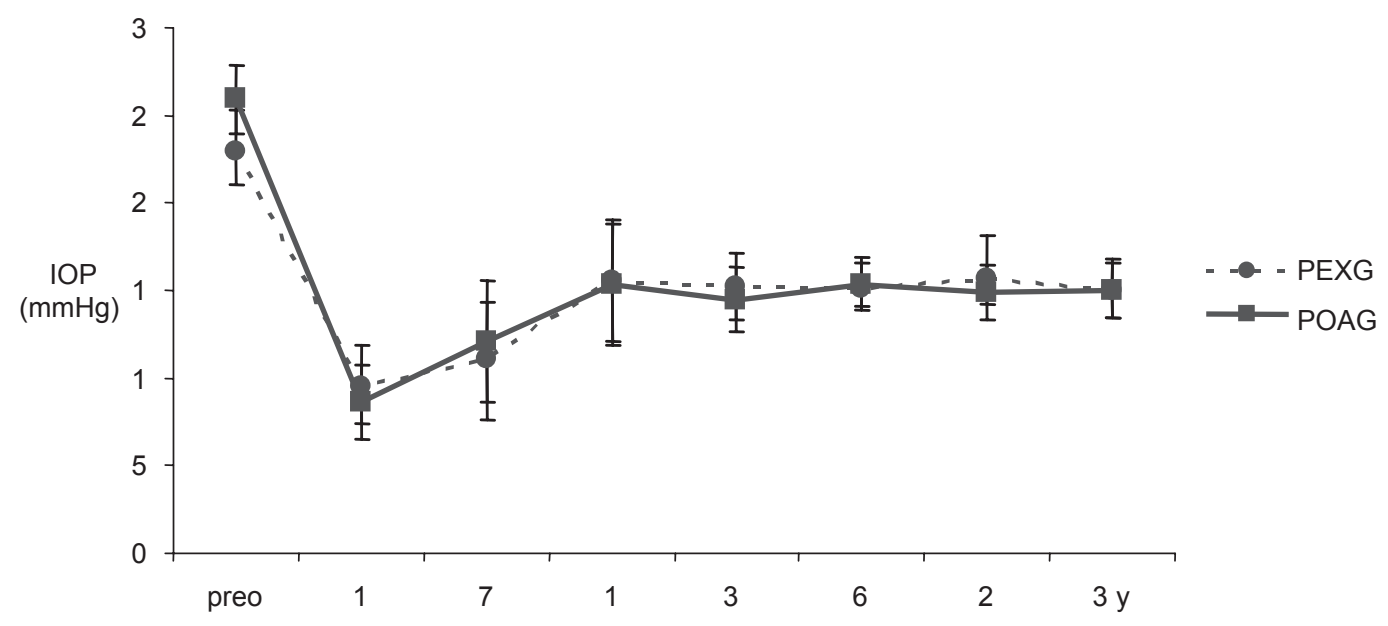

Figure 2a One-site phacotrabeculectomy. Mean IOPs in the PEXG and POAG groups at all time points. Abbreviations: IOP, intraocular pressure; PEXG, pseudoexfoliation glaucoma; POAG, primary open-angle glaucoma. 


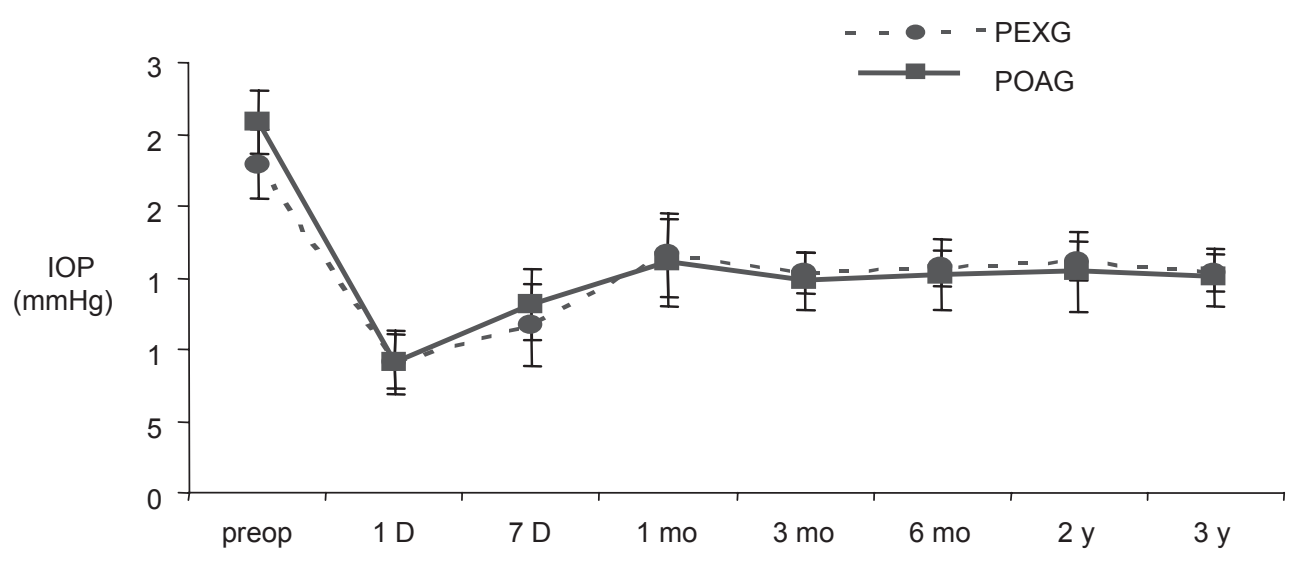

Figure 2b Two-site phacotrabeculectomy. Mean IOPs in the PEXG and POAG groups at all time points.

Abbreviations: IOP, intraocular pressure; PEXG, pseudoexfoliation glaucoma; POAG, primary open-angle glaucoma.

patients from the PEXG group (23 patients) compared to the POAG group (15 patients) had IOP $>18 \mathrm{mmHg} 2-3$ weeks postoperatively and received 5-FU injections $(p>0.05)$. We speculated that this more aggressive treatment (use of antimetabolites) of these patients at the early postoperative period had as a result more effective bleb filtration and possibly better control of their IOP in long term (three years follow up). In that way the whole group of patients with PEXG patients achieved excellent results (mean IOP, number of patients requiring antiglaucoma medications and mean number of antiglaucoma medications), similar to POAG patients. Concerning the number of patients receiving 5-FU injections we did not observe statistically significant differences between one- and two-site groups independent of the type of glaucoma they had, which was in accordance with a previous study. ${ }^{14}$

The good results obtained with combined procedure in both treated glaucoma groups, imply that either technique may be used to handle the problem of concurrent cataract and glaucoma in many patients. Factors that may favor a one-site approach are faster surgical time and reduced number of corneal incisions. However, there are situations in

Table 6 Eyes not requiring antiglaucoma medication by the end of the follow up (three years)

\begin{tabular}{llll}
\hline & POAG & PEXG & P \\
\hline One-site phacotrabeculectomy & $\mathrm{II} / 24(45.8 \%)$ & $6 / 23(26.1 \%)$ & 0.159 \\
Two-site phacotrabeculectomy & $9 / 23(39.1 \%)$ & $7 / 23(30.4 \%)$ & 0.536 \\
Total & $20 / 47(42.6 \%)$ & $13 / 46(28.3 \%)$ & 0.150 \\
\hline
\end{tabular}

Abbreviations: PEXG, pseudoexfoliation glaucoma; POAG, primary open-angle glaucoma.

Note: Chi square test was performed. which a two-site approach may be indicated such as surgeon familiarity with temporal phacoemulsification, orbital anatomy and limited superior access or cases of conjunctival scarring limiting the location of the trabeculectomy to a site that would make phacoemulsification through the same incision difficult.

In conclusion, both approaches can be performed in patients with POAG and PEXG with the expectation of improved VA and IOP control.

\section{Disclosure}

The authors report no conflicts of interest in this work.

\section{References}

1. Carlson DW, Alward WL, Barad JP, et al. A randomized study of mitomycin augmentation in combined phacoemulsification and trabeculectomy. Ophthalmology. 1997;104:719-724.

2. Cohen JS, Greff LJ, Novack GD, et al. A placebocontrolled, doublemasked evaluation of mitomycin C in combined glaucoma and cataract procedures. Ophthalmology. 1996;103:1934-1942.

3. Gandolfi SA, Vecchi M. 5-fluorouracil in combined trabeculectomy and clear-corneal phacoemulsification with posterior chamber intraocular lens implantation. Ophthalmology. 1997;104:181-186.

4. Vass C, Menapace R. Surgical strategies in patients with combined cataract and glaucoma. Curr Opin Ophthalmol. 2004;15:61-66.

5. Borggrefe J, Lieb W, Grehn F. A prospective randomized comparison of two techniques of combined cataract-glaucoma surgery. Graefes Arch Clin Exp Ophthalmol. 1999;237:887-892.

6. Mandic Z, Ivekovic R, Petric I, et al. Glaucoma triple procedure: a one-site vs a two-site approach. Coll Antropol. 2000;24:367-371.

7. el Sayyad F, Helal M, El-Maghraby A, et al. One-site versus 2-site phacotrabeculectomy; a randomized study. J Cataract Refract Surg. 1999;25:77-82.

8. Bradford J, Shingleton M, Richard S, et al. Comparison of 1-site versus 2-site phacotrabeculectomy. J Cataract Refract Surg. 2006;32: 799-802.

9. Rossetti L, Bucci L, Miglior S, et al. Temporal corneal phacoemulsification combined with separate-incision superior trabeculectomy vs standard phacotrabeculectomy; a comparative study. Acta Ophthalmol Scand Suppl. 1997;224:39. 
10. Wyse T, Meyer M, Ruderman JM, et al. Combined trabeculectomy and phacoemulsification; a one-site vs a two-site approach. Am J Ophthalmol. 1998;125:334-339.

11. el Sayyad F, Helal M, El-Maghraby A, et al. Comparison of 1-site versus 2-site phacotrabeculectomy. J Cataract Refract Surg. 2006;32:799-802.

12. Landa G, Pollack A, Marcovich A, et al. Results of combined phacoemulsification and trabeculectomy with mitomycin $\mathrm{C}$ in pseudoexfoliation versus non-pseudoexfoliation glaucoma. Graefes Arch Clin Exp Ophthalmol. 2005;243:1236-1240.
13. Buys YM, Chipman ML, Zack B, et al. Prospective randomized comparison of one- versus two-site phacotrabeculectomy. Ophthalmology. 2008;115(7):1130-1133.e1.

14. Cotran PR, Roh S, McGwin G. Randomized comparison of 1-site and 2-site phacotrabeculectomy with 3-year follow-up. Ophthalmology. 2008; 115:447-454. 
